Article

\title{
Functional Contributions of Positive Charges in the Pore-Lining Helix 3 of the Bordetella pertussis CyaA-Hemolysin to Hemolytic Activity and Ion-Channel Opening
}

\author{
Chattip Kurehong ${ }^{1}$, Chalermpol Kanchanawarin ${ }^{2}$, Busaba Powthongchin ${ }^{3}$, Panchika Prangkio ${ }^{4}$, \\ Gerd Katzenmeier ${ }^{1}$ and Chanan Angsuthanasombat ${ }^{1,5, *}$ \\ 1 Bacterial Protein Toxin Research Cluster, Institute of Molecular Biosciences, Mahidol University, \\ Salaya Campus, Nakornpathom 73170, Thailand; chattipk@gmail.com (C.K.); \\ katzenmeier.ger@mahidol.ac.th (G.K.) \\ 2 Laboratory of Theoretical and Computational Biophysics, Department of Physics, Faculty of Science, \\ Kasetsart University, Bangkok 10900, Thailand; fscicpk@ku.ac.th \\ 3 Department of Biopharmacy, Faculty of Pharmacy, Silpakorn University, Nakornpathom 73000, Thailand; \\ p.busaba.su@gmail.com \\ 4 Division of Biochemistry and Biochemical Technology, Department of Chemistry, Faculty of Science, \\ Chiang Mai University, Chiang Mai 50200, Thailand; panchikap@gmail.com \\ 5 Laboratory of Molecular Biophysics and Chemical Biology, Biophysics Institute for Research and \\ Development (BIRD), Bangkok 10160, Thailand \\ * Correspondence: chanan.ang@mahidol.ac.th; Tel.: +66-2441-9003 (ext. 1237); Fax: +66-2441-9906
}

Academic Editor: Shin-ichi Miyoshi

Received: 11 February 2017; Accepted: 10 March 2017; Published: 16 March 2017

\begin{abstract}
The Bordetella pertussis CyaA-hemolysin (CyaA-Hly) domain was previously demonstrated to be an important determinant for hemolysis against target erythrocytes and ion-channel formation in planar lipid bilayers (PLBs). Here, net-charge variations in the pore-lining helix of thirteen related RTX cytolysins including CyaA-Hly were revealed by amino acid sequence alignments, reflecting their different degrees of hemolytic activity. To analyze possible functional effects of net-charge alterations on hemolytic activity and channel formation of CyaA-Hly, specific mutations were made at $\mathrm{Gln}^{574}$ or Glu ${ }^{581}$ in its pore-lining $\alpha 3$ of which both residues are highly conserved Lys in the three highly active RTX cytolysins (i.e., Escherichia coli $\alpha$-hemolysin, Actinobacillus pleuropneumoniae toxin, and Aggregatibacter actinomycetemcomitans leukotoxin). All six constructed CyaA-Hly mutants that were over-expressed in E. coli as $126 \mathrm{kDa}$ His-tagged soluble proteins were successfully purified via immobilized $\mathrm{Ni}^{2+}$-affinity chromatography. Both positive-charge substitutions (Q574K, Q574R, E581K, E581R) and negative-charge elimination (E581Q) appeared to increase the kinetics of toxin-induced hemolysis while the substitution with a negatively-charged side-chain (Q574E) completely abolished its hemolytic activity. When incorporated into PLBs under symmetrical conditions (1.0 M KCl, pH 7.4), all five mutant toxins with the increased hemolytic activity produced clearly-resolved single channels with higher open probability and longer lifetime than the wild-type toxin, albeit with a half decrease in their maximum conductance. Molecular dynamics simulations for $50 \mathrm{~ns}$ of a trimeric CyaA-Hly pore model comprising three $\alpha 2$-loop- $\alpha 3$ transmembrane hairpins revealed a significant role of the positive charge at both target positions in the structural stability and enlarged diameter of the simulated pore. Altogether, our present data have disclosed functional contributions of positively-charged side-chains substituted at positions $\mathrm{Gln}^{574}$ and $\mathrm{Glu}^{581}$ in the pore-lining $\alpha 3$ to the enhanced hemolytic activity and ion-channel opening of CyaA-Hly that actually mimics the highly-active RTX (repeat-in-toxin) cytolysins.
\end{abstract}

Keywords: Bordetella pertussis; CyaA-hemolysin; MD simulations; channel-open lifetime; pore-lining helix; RTX cytolysin; trimeric pore 


\section{Introduction}

Bordetella pertussis is a causative agent of human whooping cough (also known as "pertussis") which has now re-emerged globally as a consequence of pathogen adaptation to vaccination and/or waning protection from acellular pertussis (aP) vaccines [1-3]. An improved version of $\mathrm{aP}$ vaccines has been made by including additional virulence factors, e.g., adenylate cyclase-hemolysin toxin (CyaA, $180 \mathrm{kDa}$ ), which was previously shown to be an effective protective antigen in mice [4,5]. CyaA is an RTX (Repeat-in-ToXin) cytolysin that facilitates respiratory tract colonization of B. pertussis by impairing function of host macrophages [6]. Very recently, we have successfully generated CyaA-specific $\mathrm{VH} / \mathrm{V}_{\mathrm{H}} \mathrm{H}$ nanobodies that could have a potential for test-driven development of a ready-to-use therapeutic in passive immunization for mitigation of disease severity [7].

The $\sim 180 \mathrm{kDa}$ CyaA toxin is synthesized as a bi-functional polypeptide (1706 residues) of which the $N$-terminal adenylate cyclase domain (AC, 400 residues), complementary to the C-terminal pore-forming/hemolysin domain (Hly, 1306 residues), has made CyaA different from other typical RTX cytolysins [8] (see Figure 1A). In the CyaA-Hly domain, there is a $100 \mathrm{kDa}$ RTX segment containing $\sim 40$ repeats of Gly-Asp-rich nonapeptides (X-U-X-Gly-Gly-X-Gly-X-Asp, X for any amino acid and U for large hydrophobic residues) that serves as $\mathrm{Ca}^{2+}$-binding sites [9-11]. The requirement of $\mathrm{Ca}^{2+}$ for either structural stability against proteolytic degradation [11] or proper folding into $\beta$-rolls of the RTX sub-domain for accelerating toxin secretion [12] has been evidently demonstrated.

For biological activity, CyaA and/or CyaA-Hly needs a palmitoyl group to be added at Lys ${ }^{983}$ by acyltransferase (CyaC, $21 \mathrm{kDa})[13,14]$ and is subsequently secreted into the surrounding environment where it selectively binds to the $\alpha_{M} \beta_{2}$ integrin receptor (also known as CD11b/CD18) on human macrophages via the RTX segment [15]. Upon such specific receptor-binding, the $\sim 130$ kDa CyaA-Hly domain would mediate translocation of the $\sim 40 \mathrm{kDa}$ catalytic AC domain into the cytosol of target cells causing apoptotic cell death [16]. The attached palmitoyl moiety was suggested to increase membrane affinity of the CyaA toxin needed for target cell attachment by serving either as a mediator of membrane associations or a determinant for receptor-toxin interactions [17]. However, we have recently demonstrated that such palmitoylation at Lys ${ }^{983}$ is not required for the binding of CyaA-Hly to target erythrocyte membranes, but rather needed for stabilizing CyaA-Hly-induced pores/channels [18]. Several studies clearly shown that, without the AC part, the CyaA-Hly domain can independently exert hemolytic activity against sheep erythrocytes that lack the CD11b/CD18 receptor, suggesting an alternative mechanism of target cell recognition via the RTX subdomain [19-22]. Nevertheless, the exact molecular mechanism of translocation of the AC domain across target cell membranes still needs to be elucidated. Of particular interest, pore formation by the Hly domain is considered as part of the critical step that mediates receptor binding and internalization of the AC domain into the cell cytosol. Better understanding of the behavior of the CyaA/CyaA-Hly-induced pores/channels in model membranes would provide more insight into the structural and biophysical characteristics of the toxin regarding the intoxication process.

Thus far, there is no three-dimensional structure of RTX cytolysins available. We have, therefore, prefigured the $N$-terminal hydrophobic segment (HP, residues 500-700) of CyaA-Hly that could adopt five putative transmembrane helices (i.e., $\alpha 1_{(500-522)}, \alpha 2_{(529-550)}, \alpha 3_{(570-93)}, \alpha 4_{(602-627)}$, and $\left.\alpha 5_{(678-698)}\right)$ of which $\alpha 2$ and $\alpha 3$ could be the pore-lining constituent participating in hemolytic activity [23]. Our recent studies via single-Ala substitutions in $\alpha 2$ revealed that the Gly ${ }^{530}$ Gly $^{533}$ _Gly ${ }^{537}$ cluster, which is arranged as a GXXGXXXG motif, plays a crucial role in CyaA-Hly-induced hemolysis, presumably involved in helix-helix association of the pore-forming helices [24]. We have also demonstrated that two key residues in $\alpha 3$, i.e., $\mathrm{Glu}^{570}$ and $\mathrm{Glu}^{581}$, are important for toxin activity, conceivably lining the lumen cavity so as to regulate the toxin-induced pore functions [25]. Nonetheless, more structural details of membrane-pore formation by CyaA-Hly still need to be intensively investigated. In the present study, a role of positive charges encompassing the $\alpha 3$-pore-lining region was investigated through net-charged alterations particularly at the two selected positions (i.e., Gln ${ }^{574}$ and $\mathrm{Glu}^{581}$ ). We demonstrate here that replacements with a positively-charged residue at either Gln ${ }^{574}$ or Glu ${ }^{581}$ 
significantly enhance hemolytic activity of the mutant toxins, consistent with an increase in the lifetime of open channels induced by each mutant toxin in PLBs. Additionally, molecular dynamics (MD) simulations of a CyaA-Hly trimeric pore model (based on our previous quantitative hemolysis assays [26]) in lipid membranes revealed a positive impact of such net-charge alterations, suggesting a significant contribution of positively-charged side-chains at positions $\mathrm{Gln}^{574}$ and $\mathrm{Glu}^{581}$ in $\alpha 3$ to CyaA-Hly functional activity.

A

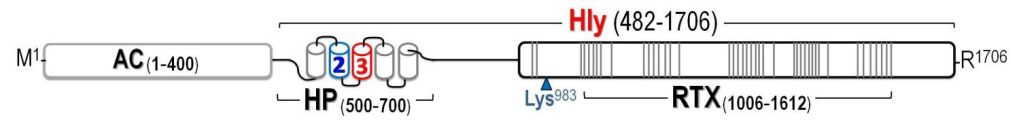

B

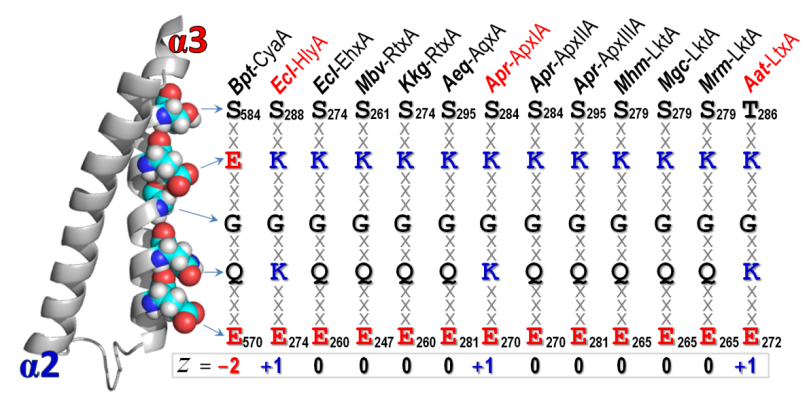

C

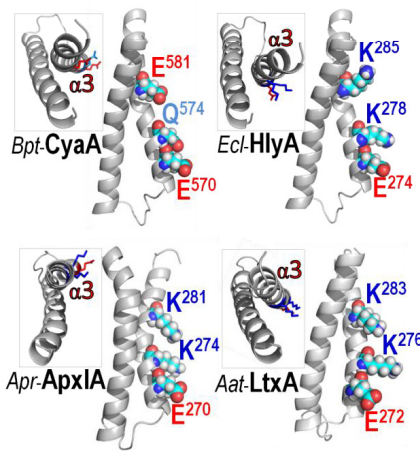

Figure 1. (A) (Above) Schematic representation of CyaA showing adenylate cyclase (AC) domain and hemolysin (Hly) domain which contains the hydrophobic (HP) region, palmitoylation site (Lys ${ }^{983}$ ) and the RTX region. Five putative helices in the HP region are indicated by cylinders where $\alpha 2$ and $\alpha 3$, putative membrane-inserting constituents were marked in blue and red, respectively. Each line in the RTX region represents each repeat of (X-U-X-Gly-Gly-X-Gly-X-Asp, $X$ for any amino acid and $U$ for large hydrophobic residues); (B) Left panel: The homology-based model of the CyaA-Hly $\alpha 2-\alpha 3$ hairpin. Side chains at pore-lining region of $\alpha 3$ are shown as van der Waals (vdW) spheres and colored according to atoms: cyan, white, red, and blue for $\mathrm{C}, \mathrm{H}, \mathrm{O}$, and N, respectively. Right panel: Conserved amino acids at pore-lining regions of thirteen related RTX cytolysins, as mentioned earlier in [25]. The side-chains are highlighted in bold letter and the charged residues are colored in red for negative and blue for positive; and (C) Side view of the hairpins of CyaA-Hly and three highly-active RTX cytolysins, i.e., HlyA from E. coli, ApxIA from Actinobacillus pleuropneumoniae and LtxA from Aggregatibacter actinomycetemcomitans, showing three key side chains at $\mathrm{Glu}^{570} / \mathrm{Gln}^{574} / \mathrm{Glu}^{581}$ (for CyaA-Hly) and Glu/Lys/Lys (for HlyA, ApxIA, and LtxA) patches. Inset: Top view of individual hairpins. Amino acids are colored according to their charged/polar properties (red is negatively-charged, blue is positively-charged, and light-blue is $\mathrm{N}$-containing polar uncharged) with $\mathrm{H}$ atoms omitted. 


\section{Results and Discussion}

\subsection{Net-Charge Variations in Pore-Lining Helix of RTX Cytolysins}

The HP region of several RTX cytolysins including CyaA has been shown to play a key role in membrane-pore formation $[8,23,27]$. We previously demonstrated that two putative transmembrane helices ( $\alpha 2$ and $\alpha 3)$ in the CyaA-Hly domain are crucially involved in hemolytic activity [23-25]. Here, as revealed from the Kyte-Doolittle-hydropathy analysis [28], four potential transmembrane segments corresponding to $\alpha 2-\alpha 5$ of CyaA-Hly can be identified in the HP region of among thirteen related RTX cytolysins including three highly active members, i.e., Escherichia coli $\alpha$-hemolysin (HlyA), Actinobacillus pleuropneumoniae toxin (ApxIA), and Aggregatibacter actinomycetemcomitans leukotoxin (LtxA) (see Supplementary Figure S1). This implies that the CyaA-Hly $\alpha 2 / \alpha 3$ homologues of these related RTX cytolysins could also play an important role in membrane insertion and pore formation. Of particular interest, the $\alpha 3$ homologues that contain highly conserved uncharged polar and charged side-chains (Figure 1B) could conceivably be part of the pore-lining constituent facing the lumen cavity.

Further analysis via multiple alignments of corresponding CyaA-Hly $\alpha 3$ sequences among the thirteen RTX cytolysins shown in Figure 1B revealed variations in their net charge $(z)$ that can be categorized into three groups: (i) a negatively-charged patch $(z=-2)$ of CyaA-Hly, containing $\mathrm{Glu}^{570} / \mathrm{Gln}^{574} / \mathrm{Glu}^{581}$; (ii) a positively-charged patch $(z=+1)$ of HlyA, ApxIA, and LtxA, containing Glu/Lys/Lys; and (iii) a neutral patch $(z=0)$ of nine other RTX cytolysins, containing Glu/Gln/Lys. It is important to note that the group with $z=+1$ (i.e., Ecl-HlyA, Apr-ApxIA, and Aat-LtxA) exhibited a very strong hemolytic activity while the group with $z=-2$ (i.e., CyaA-Hly) displayed a relatively weak activity [6,29-31]. Thus, such surface net-charge variations in the pore-lining helix of these RTX cytolysins could reflect their different degrees of hemolytic/pore-forming activities.

\subsection{Effects of Net-Charge Alterations at Gln ${ }^{574}$ and Glu ${ }^{581}$ on CyaA-Hly Hemolysis}

To test whether a positive net-charge covering the pore-lining region is essentially associated with a strong hemolytic activity of RTX cytolysins, we carried out net-charge alterations in the negatively-charged patch $(z=-2)$ of CyaA-Hly as a study model. It is worth mentioning that the negatively-charged $\mathrm{Glu}^{570}$ which is one of the patch constituents (Glu ${ }^{570} / \mathrm{Gln}^{574} / \mathrm{Glu}^{581}$ ) in CyaA-Hly $\alpha 3$ and is highly conserved throughout the RTX cytolysin family, was previously demonstrated to be important for hemolytic activity of CyaA-Hly [25]. We, therefore, focused on only two other positions, i.e., $\mathrm{Gln}^{574}$ and $\mathrm{Glu}^{581}$, of which both positions in all the three highly-active RTX cytolysins (i.e., Ecl-HlyA, Apr-ApxIA, and Aat-LtxA) have a preference for positively-charged Lys (see Figure 1B,C). Although, to our knowledge, these two highly-conserved Lys side-chains have not yet been characterized for their functional significance, they might possibly contribute to the high hemolytic activity of Ecl-HlyA, Apr-ApxIA, and Aat-LtxA. Herein, a total of six single-substituted mutants (Q574K, Q574R, Q574E, E581K, E581R, and E581Q) at both Gln ${ }^{574}$ and $\mathrm{Glu}^{581}$ were constructed corresponding to either charge alteration or elimination. Upon induction with isopropyl- $\beta$-D-thiogalactopyranoside (IPTG), all verified CyaA-Hly mutant clones were able to express $6 \times$ His-tagged soluble proteins $\left(\mathrm{CyaA}-\mathrm{Hly} / \mathrm{H}_{6}\right)$ of $126 \mathrm{kDa}$ at high levels comparable to the wild-type clone (see Supplementary Figure S2). Further analysis by Western blotting confirmed that all the CyaA-Hly $/ \mathrm{H}_{6}$ mutant toxins were recognized by both anti-His and anti-RTX antisera, verifying the presence of a $6 \times$ His-affinity tag as well as its RTX identity. Subsequently, the wild-type and mutant toxins were successfully purified using the same immobilized $\mathrm{Ni}^{2+}$-affinity chromatographic conditions, suggesting that they all preserved their $\mathrm{Ni}^{2+}$-NTA binding characteristics. As analyzed by SDS-PAGE, the $126 \mathrm{kDa}$ protein band with $>90 \%$ purity of all the CyaA-Hly $/ \mathrm{H}_{6}$ mutants was efficiently obtained as good as the wild-type toxin via a stepwise elution with $75 \mathrm{mM}$ imidazole (Figure 2 subset). 


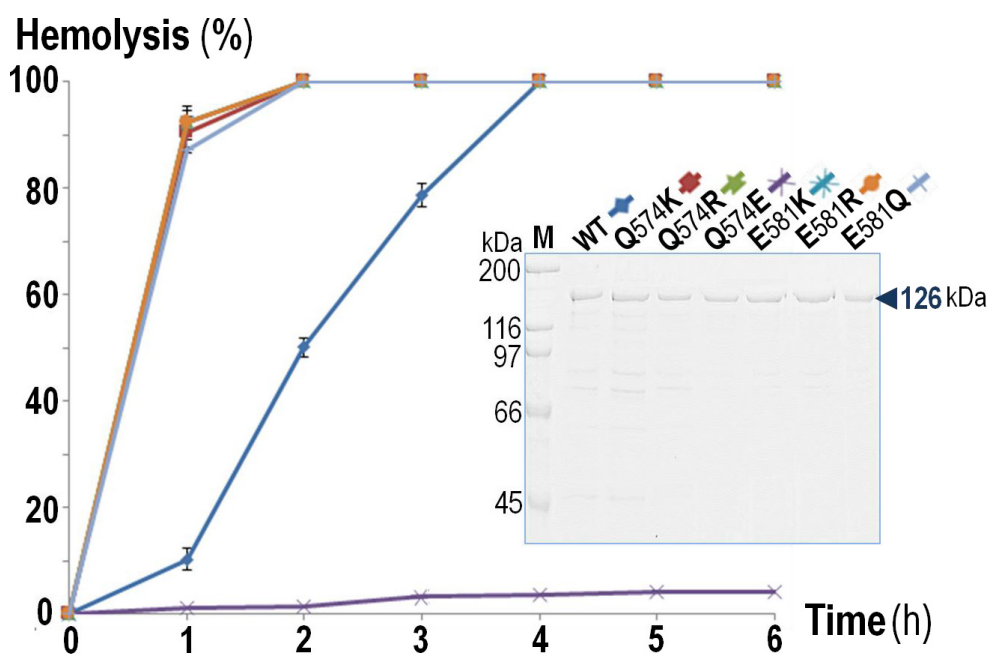

Figure 2. Time-course of hemolytic activity against sheep erythrocytes of individual purified toxins $(10 \mu \mathrm{g} / \mathrm{mL})$ as indicated. The control for $100 \%$ hemolysis is susceptible sheep erythrocytes with respect to a complete lysis when treated with $0.1 \%$ Triton X-100. Subset: SDS-PAGE (10\% gel) of the $\mathrm{Ni}^{2+}-\mathrm{NTA}$ purified CyaA-Hly toxins, both wild-type (WT) and mutants (Q574K, Q574R, Q574E, E581K, E581R, and E581Q). Bands of CyaA-Hly toxins $126 \mathrm{kDa}$ in length are marked by an arrow.

To further determine an effect of these net-charge alterations on CyaA-Hly hemolysis, we tested all individual mutant toxins for their relative hemolytic activity against susceptible sheep erythrocytes. The hemolysis data shown in Figure 2 reveal that the purified mutant toxins obtained via positive-charge substitutions (Q574K, Q574R, E581K, E581R) or the negative-charge elimination (E581Q) could exhibit a significantly enhanced activity as they cause complete hemolysis against sheep erythrocytes within $2 \mathrm{~h}$, much faster than the wild-type toxin, which required up to $4 \mathrm{~h}$. However, the negative-charge substitution at $\mathrm{Gl}^{574}$ (Q574E) completely eliminated toxin hemolytic activity. These results supported the preference of a positive charge at either position $\left(\mathrm{Gln}^{574}\right.$ or $\left.\mathrm{Glu}^{581}\right)$ facing the pore interior of which its net charge has changed from -2 to -1 for Q574K and Q574R mutants and from -2 to 0 for E581K and E581R mutants. Moreover, the non-preference of the negative charge was confirmed by the enhanced activity of the E581Q mutant ( $z$ decreased from -2 to -1 ) and the impaired activity of the Q574E mutant ( $z$ increased from -2 to -3 ). Loss in hemolytic activity of the Q574E mutant might be due to a local structure distortion caused by the introduced negatively-charged Glu that faces the same side and is close enough to generate charge-charge repulsion with the key functional residue-Glu ${ }^{570}$ (see Figure 1C). Accordingly, our results demonstrated that specific mutations at $\mathrm{Gln}^{574}$ or Glu ${ }^{581}$ in CyaA-Hly $\alpha 3$ for resembling the positively-charged patch $(z=+1)$ of the highly-active RTX cytolysins (i.e., Ecl-HlyA, Apr-ApxIA and Aat-LtxA) could improve lytic pore-forming efficiency of CyaA-Hly toward sheep erythrocytes. Thus, these results support an important contribution of a positive net-charge covering the pore-lining region of CyaA-Hly $\alpha 3$ to an enhancing effect on toxin hemolytic activity.

\subsection{Ion-Channel Characteristics Formed by CyaA-Hly Mutant Toxins with Enhanced Hemolytic Activity}

Experiments were further conducted to assess channel-forming properties of the purified CyaA-Hly $/ \mathrm{H}_{6}$ mutants in comparison with that of the wild-type toxin. By incorporation into PLBs under symmetrical ionic conditions (at $1 \mathrm{M} \mathrm{KCl}, 10 \mathrm{mM}$ HEPES, $\mathrm{pH} 7.4$ ), the wild-type toxin could cause ion channels to open with a maximum conductance $\left(g_{\mathrm{K}}\right)$ of $36.4 \pm 1.7 \mathrm{pS}$, as shown by representative current traces (Figure 3A). However, the positive-charge substituted mutants with enhanced hemolytic activity, i.e., Q574K, Q574R, E581K, and E581R, could induce the channels to open with approximately a half decrease in $g_{\mathrm{K}}(17.1 \pm 0.2,22.0 \pm 0.3,19.3 \pm 0.2$, and $16.4 \pm 0.6 \mathrm{pS}$, respectively), except for 
the E581Q mutant of which $g_{\mathrm{K}}$ remained relatively unchanged (34.7 $\pm 0.4 \mathrm{pS}$ ) (see Figure 3B and Table 1). It was also found that the hemolytically-inactive Q574E mutant was unable to produce channel conductance in PLBs, confirming the non-preference of the negatively-charged side-chain at this position.

A

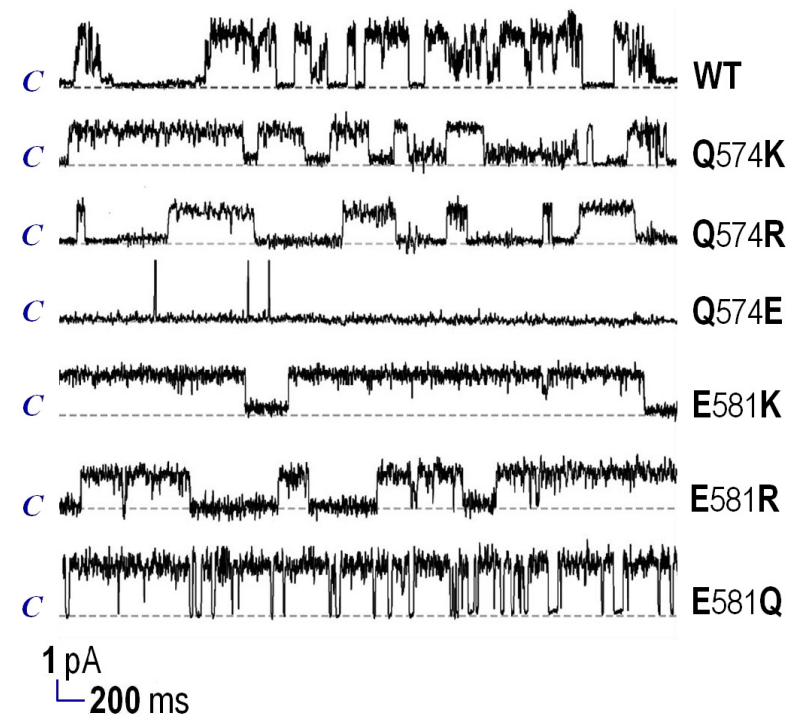

B

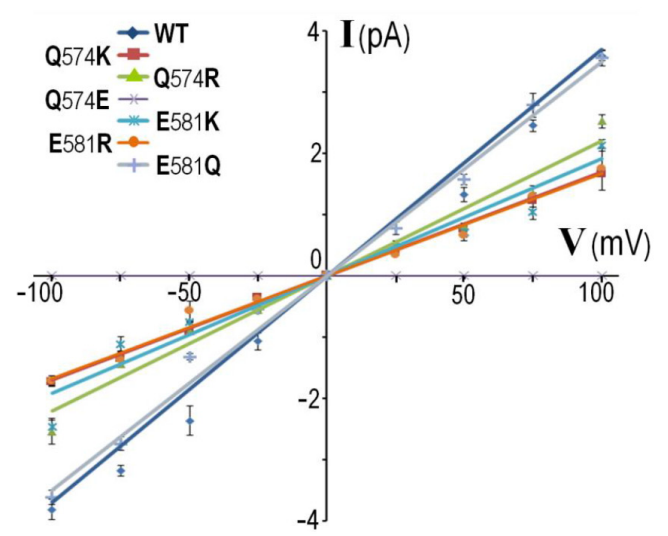

Figure 3. Ion-channel properties of CyaA-Hly wild-type (WT) and its mutant toxins $(1 \mu \mathrm{g} / \mathrm{mL})$. (A) Representative current traces were recorded at $100 \mathrm{mV}$ holding potential. The closed stage level of channels is denoted by the letter $c$. Vertical and horizontal bars indicate measured current-time scales, respectively; and (B) current-voltage relations of all single channels formed by WT and its mutant toxins were recorded under symmetric conditions.

Remarkably, the channels induced by these positive-charge substituted mutants: Q574K, Q574R, E581K, and E581R, appeared to have significant longer open lifetimes (i.e., 1620, 2980, 1920 and $2500 \mathrm{~ms}$, respectively) than that induced by wild-type toxin ( 235 ms) while the E581Q mutant's channel exhibited a slightly shorter lifetime ( $\sim 150 \mathrm{~ms}$ ) (see Table 1$)$. In addition, all the active mutants showed a marked increase in the open channel probability $(0.78,0.53,0.71,0.82$, and 0.76 for Q574K, Q574R, E581K, E581R, and E581Q, respectively) compared to the wild-type toxin (0.29). It is possible that the channel interior with less negative charge could prolong open-channel lifetimes and increase open-channel probability, conceivably accounting for their enhanced hemolytic activity. As revealed in certain ion channels and transporters, the preference of charged residues at particular locations inside the pores can affect their structural arrangement through interactions between neighboring oppositely-charged residues [32-34]. However, all four hemolytically-active mutants substituted 
with a positive charge (i.e., Q574K, Q574R, E581K, and E581R) appeared to produce single-channels with about a half decrease in maximum conductance compared to the wild-type and E581Q mutant toxins as demonstrated above (see Figure 3). Therefore, an introduced positively-charged side-chain at these two positions could perhaps disturb cation-conducting ability of CyaA-Hly channels, implying the disparate manners of CyaA-Hly in regulating between its hemolytic pore formation and ion-conduction.

Table 1. Channel characteristics of CyaA-Hly wild type and mutants.

\begin{tabular}{cccccc}
\hline Toxins $^{\mathbf{a}}$ & $z^{\mathbf{b}}$ & Hemolytic Activity $^{\mathbf{c}}$ & $g_{\mathbf{K}}(\mathbf{p S})^{\mathbf{d}}$ & ${\text { Open Lifetime }(\mathbf{m s})^{\mathbf{e}}}$ & Open Probability $^{\mathbf{f}}$ \\
\hline WT & -6 & $10.1 \pm 1.1 \%$ & $36.1 \pm 1.7$ & $236 \pm 1$ & 0.29 \\
Q574K & -3 & $35.9 \pm 1.0 \%$ & $17.1 \pm 0.2$ & $1620 \pm 12$ & 0.78 \\
Q574R & -3 & $36.6 \pm 0.7 \%$ & $22.0 \pm 0.3$ & $2980 \pm 20$ & 0.53 \\
Q574E & -9 & $1.6 \pm 0.1 \%$ & $-\mathrm{g}$ & $-\mathrm{g}$ & $-\mathrm{g}$ \\
E581K & 0 & $35.1 \pm 1.1 \%$ & $19.3 \pm 0.2$ & $1920 \pm 16$ & 0.71 \\
E581R & 0 & $30.0 \pm 1.0 \%$ & $16.4 \pm 0.6$ & $2500 \pm 14$ & 0.82 \\
E581Q & -3 & $32.3 \pm 1.0 \%$ & $34.7 \pm 0.4$ & $147 \pm 2$ & 0.76 \\
\hline
\end{tabular}

${ }^{a} \mathrm{Ni}^{2+}$-NTA purified toxins; ${ }^{\mathrm{b}}$ Surface charge of interior pore in trimeric model; ${ }^{\mathrm{c}} \%$ Hemolytic activities of CyaA-Hly toxins $(1 \mu \mathrm{g} / \mathrm{mL})$ against sheep erythrocytes at $6 \mathrm{~h}$; ${ }^{\mathrm{d}}$ The single channel conductance of $1 \mu \mathrm{g} / \mathrm{mL}$ toxins recorded in $1 \mathrm{M} \mathrm{KCl}$ solution (pH 7.4), and presented as mean \pm SEM from three independent experiments; ${ }^{\mathrm{e}}$ The open channel lifetime was calculated from $\sim 100$ open events in $1 \mathrm{M} \mathrm{KCl}$ solution (pH 7.4) at $100 \mathrm{mV}$ holding potential from equation; $\mathrm{N}(t) / \mathrm{N}(0)=\exp (-t / \tau) ;{ }^{\mathrm{f}}$ Open probability was calculated from $\sim 100$ open events in $1 \mathrm{M} \mathrm{KCl}$ solution (pH 7.4) at $100 \mathrm{mV}$ holding potential from equation $P_{\text {open }}=t_{\text {open }} /\left(t_{\text {open }}+t_{\text {closed }}\right) ;{ }^{g}$ The event cannot be observed.

\subsection{Trimeric Pore Models and Effects of Net-Charge Alterations on Their Structural Dynamics}

To gain more insights into the architecture of the CyaA-Hly pore/channel, we constructed a plausible 3D-modeled pore by docking three copies of the $\alpha 2$-loop- $\alpha 3$ hairpin, based on osmotic protection experiments, which estimated the CyaA-induced pore diameter to be approximately 6-8 $\AA$ [35], and our previous quantitative hemolysis assays, which revealed that the toxin could cooperatively form a trimeric pore with a Hill coefficient of $\sim 3$ [26]. This similar finding could be observed in some other bacterial protein toxins from entirely different family, for instance, Bacillus thuringiensis Cry4Ba insecticidal protein, which was also demonstrated to form a trimeric pore structure [36,37]. These two pore-forming toxins potentially share a similar pore structure and mechanism of pore formation, although they have no sequence similarities outside of their pore-lining constituents. Thus, nature seems to have developed an analogous design for the two toxin-induced pores/channels by different evolutionary pathways.

A possible role of the positive charge substituted at either critical position ( $\mathrm{Gln}^{574}$ or $\mathrm{Glu}^{581}$ ) conceivably located at the CyaA-Hly pore interior was subsequently characterized via 50-ns MD simulations of four individual trimeric pore-mutant models with positive-charge substitutions, Q574K, Q574R, E581K, and E581R (see Figure 4A). As further illustrated in Figure 4B, replacement with a positive charge (either Lys or Arg) at $\mathrm{Gl}^{570}$ can reduce structural fluctuation of both Q574K- and Q574R-simulated pores shown by their decreased RMSF values, especially around the intracellular pore mouth at $\mathrm{Glu}^{570}$. It is conceivable that $\mathrm{Glu}^{570}$ and Lys/Arg-substituted side-chain at $\mathrm{Gln}^{574}$ which are oriented closely facing the pore lumen could perhaps create electrostatic interactions to stabilize the pore mouth. Nevertheless, the effect on RMSF was not significant when Lys/Arg was introduced at $\mathrm{Glu}^{581}$ located rather far from $\mathrm{Glu}^{570}$ (see Figure $4 \mathrm{~B}$ inset). Interestingly, positive-charge substitutions at either $\mathrm{Gln}^{574}$ or $\mathrm{Glu}^{581}$ were both found to increase pore diameter around the intracellular entry, as presented by the averaged separation distance $(d)$ of $\mathrm{Glu}^{570}$, which is $\sim 12.2 \AA$ for the wild-type, and increases to about 16.5, 13.8, 15.3, and $17.5 \AA$ for Q574K, Q574R, E581K, and E581R mutants, respectively (see Figure 4C). The increased stability and pore size of the Lys/Arg-substituted mutant pores observed herein were consistent with their prolonged open-channel lifetimes and increased open-channel probability in the PLBs as demonstrated above (see Table 1). 
A

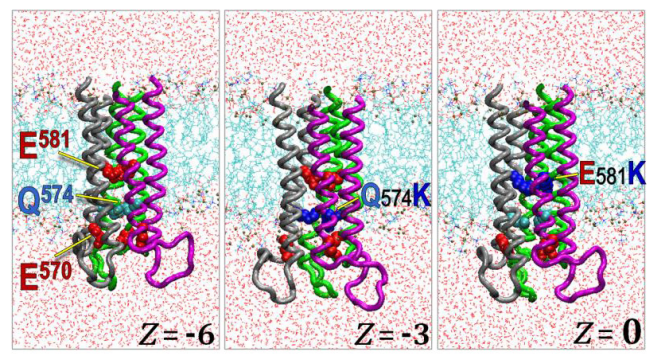

B

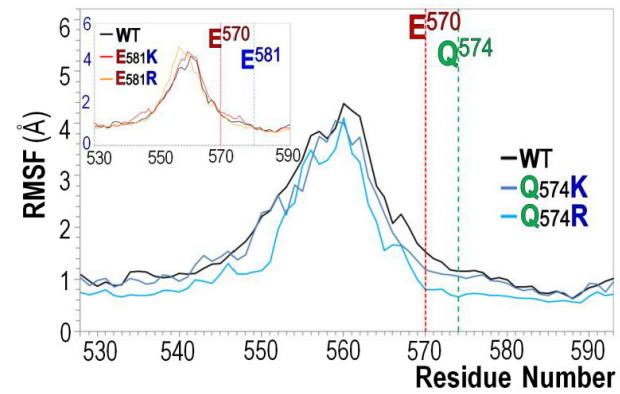

C
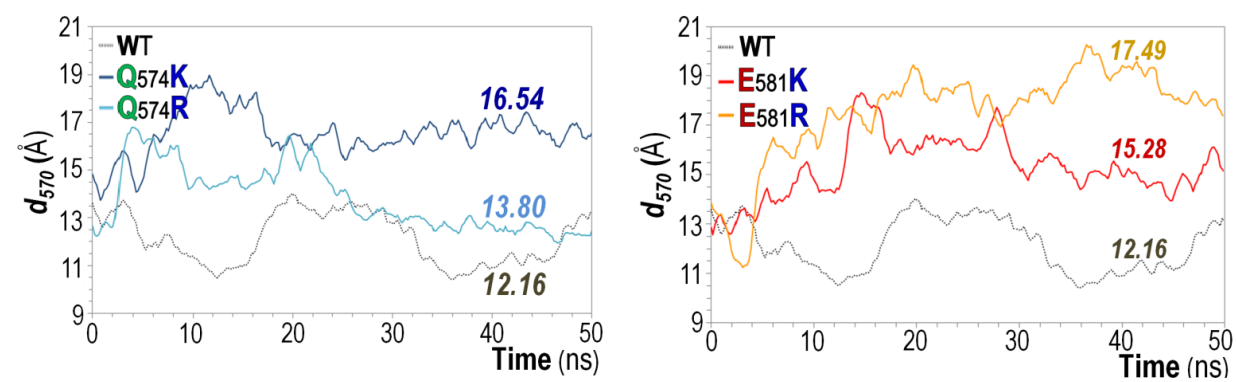

Figure 4. (A) MD simulations (50 ns) of trimeric CyaA-Hly channel models in membrane of the wild-type (WT, left) and two representative mutants; Q574K (middle) and E581K (right). Total net charges $(z)$ inside the pores are shown; (B) $C_{\alpha}$-RMSF profiles $\left(\mathrm{Arg}^{528}-\mathrm{Ala}^{593}\right.$ ) obtained from MD trajectories of 50-ns simulations of individual CyaA-Hly pore structures; and $(C)$ the average separation distance $\left(C_{\alpha}\right.$ to $\left.C_{\alpha}\right)$ between three $G^{570}{ }^{570}$ residues on the trimeric CyaA-Hly pore models versus time during 50-ns MD simulations. Left and right panels represent the systems of Gln ${ }^{574}$ and Glu ${ }^{581}$ mutants compared to the wild-type, respectively.

Taken as a whole, we have clearly demonstrated that CyaA-Hly can replicate other highly-active RTX cytolysins (e.g., Ecl-HlyA, Apr-ApxIA, and Aat-LtxA), and becomes much more hemolytically active by introducing a positively-charged side-chain at $\mathrm{Gln}^{574}$ or $\mathrm{Glu}^{581}$ (or by removing a negatively-charged side-chain at $\mathrm{Glu}^{581}$ ) in the pore-lining $\alpha 3$. The increase in the hemolytic activity of the CyaA-Hly mutants, i.e., Q574K, Q574R, E581K, E581R, and E581Q, could perhaps come from the prolonging of the pore in its open state as revealed by PLB results. Additionally, this is probably due to the larger pore diameter and more structural stability of the mutant pores as indicated in the MD simulations. Such a stable-large pore of the mutants would permeable to all ions, promoting a faster hemolysis of target erythrocytes. Moreover, introducing a negatively charged side-chain at $\mathrm{Gln}^{574}$ could give the opposite effect as the pores destabilized and became hemolytically inactive. However, in PLBs, only half conductance was observed for mutants with a positive-charge substitution, albeit being very hemolytically active against sheep erythrocytes. The explanation of this is that the introduced positive charge at whichever side-chain (i.e., $\mathrm{Gln}^{574}$ or $\mathrm{Glu}^{581}$ ) could possibly do two things, (i) it would create a positive electrostatic energy barrier that prevent cations to flow through the pore (as CyaA-induced 
channels were shown to be cation-selective [29]) and hence the reduction in conductance; and (ii) it, not only at $\mathrm{Glu}^{581}$ but also at Gln ${ }^{574}$, would stabilize the pore structure by balancing the strong electrostatic repulsions between $\mathrm{Glu}^{570}$ and $\mathrm{Glu}^{581}$ in the pore and hence the overall increase in hemolytic activity of the positive-charge substituted toxins. Thus, these findings provide insights to better understanding about membrane-pore formation of the RTX cytolysins that net-charge variations at the pore-lining region could be a factor accounting for their differences in pore-forming efficiency.

\section{Materials and Methods}

\subsection{Hydropathy Analysis and Protein Multiple Sequence Alignments}

Hydropathy plots of the HP regions from thirteen related RTX cytolysins, i.e., CyaA from $B$. pertussis (Bpt-CyaA: CAE41066), HlyA and EhxA from E. coli (Ecl-HlyA: ABE10329 and Ecl-EhxA: BAA31774), RtxA from Moraxella bovis (Mbv-RtxA: AKK84651), and Kingella kingae (Kkg-RtxA: ABK58601), AqxA from Actinobacillus equuli (Aeq-AqxA: AMM45569); ApxIA, ApxIIA and ApxIIIA from A. pleuropneumoniae (Apr-ApxIA: AAL55666, Apr-ApxIIA: AAU84700 and Apr-ApxIIIA: CAA48711), LktA from Manheimia haemolytica (Mhm-LktA: AAL13281), M. glucosida (Mgc-LktA: AAG40306), and M. ruminalis (Mrm-LktA: AAR09165) and LtxA from Aggregatibacter actinomycetemcomitans (Aat-LtxA: CAA34731), were performed as described by Kyte and Doolittle [28] with a window size of 19 residues. Multiple alignments of protein sequences were accomplished using Clustal server (Clustal W 2.0, 2007, Oxford University Press, Oxford, UK) [38].

\subsection{Homology-Based Modeling of Hairpin Structures}

Atom coordinates of $\alpha 2$-loop- $\alpha 3$ hairpin were extracted from the homology-based model of the CyaA-HP region ( $\alpha 1-\alpha 5)$, which was previously constructed [26]. The extracted CyaA-hairpin was subsequently used as a template for modeling its corresponding hairpin structures of relative RTX cytolysins via SWISS-MODEL server (2003, Oxford University Press, Oxford, UK) [39]. Modeled hairpin structures were energy-minimized using NOMAD-Ref server (2006, Oxford University Press, Oxford, UK) [40].

\subsection{Construction of Mutant Plasmids}

The pCyaAC-PF/H 6 plasmid encoding $126 \mathrm{kDa} 6 \times$ His-tagged CyaA-Hly [26] was used as a template. Complementary pairs of mutagenic primers were designed based on the CyaA-Hly gene sequence (see Supplementary Table S1). All mutant plasmids were generated by polymerase chain reaction (PCR)-based mutagenesis using high-fidelity Phusion DNA polymerase (Finnzymes, Vantaa, Finland), following the QuickChange ${ }^{\mathrm{TM}}$ mutagenesis procedure (Agilent Technologies, Santa Clara, CA, USA). Mutant plasmids were first identified by restriction endonuclease digestion and subsequently verified by DNA sequencing.

\subsection{Bacterial Culture and Toxin Expression}

E. coli strain BL21(DE3)pLysS was individually transformed with each mutant plasmids and cultured at $30{ }^{\circ} \mathrm{C}$ in Terrific broth containing ampicillin $(100 \mu \mathrm{g} / \mathrm{mL})$ and chloramphenicol $(34 \mu \mathrm{g} / \mathrm{mL})$ as previously described [26]. When the cultures reached $0.5 \mathrm{OD}_{600}$, protein expression was induced by IPTG (isopropyl- $\beta$-D-thiogalactopyranoside) at a final concentration of $0.1 \mathrm{mM}$ and then incubated for additional $4 \mathrm{hr}$.

\subsection{Toxin Purification via Immobilized Metal Affinity Chromatography}

Cultured cells were harvested $\left(6000 \times g, 4^{\circ} \mathrm{C}, 10 \mathrm{~min}\right)$, re-suspended in $50 \mathrm{mM}$ Tris- $\mathrm{HCl}(\mathrm{pH} 7.4)$ supplemented with $5 \mathrm{mM} \mathrm{CaCl} 2$ and $1 \mathrm{mM}$ protease inhibitors (phenylmethylsulfonylfluoride and 1,10-phenanthroline), and subsequently disrupted in a French Pressure Cell (10,000 psi). After centrifugation $\left(13,000 \times g, 4^{\circ} \mathrm{C}, 15 \mathrm{~min}\right)$, His-tagged soluble toxins in lysate supernatant 
were purified using the immobilized metal affinity column $\left(\mathrm{Ni}^{2+}-\mathrm{NTA}\right)$ as previously described [26]. Protein concentrations were quantified using Bradford microassay (Bio-RAD, Hercules, CA, USA) and then analysed by SDS-PAGE.

\subsection{Toxin Verification via Western Blot Analysis}

Protein toxins from SDS-PAGE were electroblotted onto a nitrocellulose membrane and then blocked with $5 \%$ non-fat milk in PBS $\left(120 \mathrm{mM} \mathrm{NaCl}, 16 \mathrm{mM} \mathrm{Na}_{2} \mathrm{HPO}_{4}, 4 \mathrm{mM} \mathrm{NaH} \mathrm{PO}_{4}, \mathrm{pH} 7.4\right)$ prior to probing with anti-RTX antibodies (1:40,000 dilution) which was raised against the $100 \mathrm{kDa}$ CyaA-RTX subdomain fragment [11]. Immune-complexes were detected with anti-rabbit IgG $2^{\circ}$ antibodies conjugated with alkaline phosphatase (AP, 1:7000 dilution; Pierce, Rockford, IL, USA) and then immuno-reactive bands were visualized by color development with 5-bromo-4-chloro-3-indolyl phosphate/nitroblue tetrazolium substrates (KPL, Gaitherburg, MD, USA). The presence of the $6 \times$ His tag was verified by probing with AP-conjugated anti-His (C-term) antibodies (1:2500 dilution; Invitrogen, Waltham, MA, USA) and BCIP/NBT color detection.

\subsection{Determination of Hemolytic Activity of Mutant Toxins}

The purified toxin $(\sim 10 \mu \mathrm{g} / \mathrm{mL})$ was assayed with sheep erythrocyte suspension $\left(5 \times 10^{8}\right.$ cells $/ \mathrm{mL}$ in $150 \mathrm{mM} \mathrm{NaCl}, 2 \mathrm{mM} \mathrm{CaCl}, 20 \mathrm{mM}$ Tris-HCl, pH 7.4) at $37^{\circ} \mathrm{C}$ for $6 \mathrm{~h}$. Non-lysed cells and debris were removed by centrifugation at $(12,000 \times g, 2 \mathrm{~min})$, and the supernatant was transferred to a 96-well microtiter plate for determining the released hemoglobin by measuring absorbance at $540 \mathrm{~nm}\left(\mathrm{OD}_{540}\right)$. Percent hemolysis for each toxin sample was calculated by [(OD $\mathrm{OD}_{540}$ sample $-\mathrm{OD}_{540}$ negative control $) /\left(\mathrm{OD}_{540}\right.$ of $100 \%$ hemolysis $-\mathrm{OD}_{540}$ negative control $\left.)\right] \times 100$. An equal amount of erythrocytes lysed with $0.1 \%$ Triton X-100 was defined as $100 \%$ hemolysis. All samples were tested in triplicate for three independent experiments as previously described [21].

\subsection{Single-Channel Analysis of Mutant Toxins via Planar Lipid Bilayers (PLBs)}

PLBs were formed by painting method $20 \mathrm{mg} / \mathrm{mL}$ of 1,2-diphytanoyl-sn-glycero-3- phosphocholine (DiPhyPC, Avanti Polar Lipids, Alabaster, AL, USA) on a 250- $\mu \mathrm{m}$ aperture in a $1 \mathrm{~mL}$-Delrin cup (Warner Instruments, Hamden, CT, USA) as previously described [26]. Toxin samples $(1 \mu \mathrm{g} / \mathrm{mL}$; prepared in $20 \mathrm{mM}$ HEPES, $\mathrm{pH} 7.4,5 \mathrm{mM} \mathrm{CaCl} 2$ ) were added into both cis and trans compartments containing recording buffer ( $1 \mathrm{M} \mathrm{KCl}, 10 \mathrm{mM}$ HEPES, $\mathrm{pH}$ 7.4). Single-channel currents were recorded with Geneclamp-500 amplifier (Axon Instruments, Sunnyvale, CA, USA) and signals (filtered at $10 \mathrm{kHz}$ ) were digitized with PCI-6221 analog-to-digital converter (National Instruments, Austin, TX, USA) using LabVIEW 7.1 software at a $50 \mathrm{kHz}$ sampling frequency. Channel conductances and lifetimes were determined from the observed current steps ( 100 open channels) at $100 \mathrm{mV}$ applied voltage.

\subsection{Trimeric Docking and Molecular Dynamics (MD) Simulations of a Modeled Pore}

A trimeric pore model of CyaA-Hly was constructed by docking three copies of the $\alpha 2$-loop- $\alpha 3$ hairpin using ZDOCK server (ZDOCK 3.0.2, 2014, Oxford University Press, Oxford, UK) [41] as previously described [24]. The model was inserted into a DMPC (1, 2 dimyristoyl-sn-glycero-3-phosphocholine) bilayer using CHARMM GUI server (2014, John Wiley \& Sons Inc., New York, NY, USA) [42] and then water and ions were added to obtain a system of CyaA-Hly trimeric pore at $150 \mathrm{mM} \mathrm{KCl} \mathrm{using} \mathrm{VMD}$ program [43]. The final system has 40,301 atoms consisting of the trimeric pore, 120 DMPC molecules, 8748 water molecules, $27 \mathrm{~K}^{+}$ions and $27 \mathrm{Cl}^{-}$ions (at $150 \mathrm{mM} \mathrm{KCl}$ ) was energetically minimized, equilibrated, and finally ran for $50 \mathrm{~ns}$ at temperature $300 \mathrm{~K}$ and pressure $1 \mathrm{~atm}$ using the NAMD program [44] and CHARMM force-fields [45] on a computer cluster with 16 CPU-cores (2.3 GHz AMD Opteron) at 2 ns per day. Full electrostatic calculation with multiple-time stepping was used with periodic boundary conditions and $2 \mathrm{fs}$ integration time. All other MD simulation parameters were set as described in [46]. The dynamical and structural properties of the trimeric pore model were analyzed from the MD trajectories using VMD program. Root mean square fluctuation (RMSF) was calculated 
from RMSF $=\sqrt{\frac{1}{T} \sum_{t=1}^{T}\left[x_{i}(t)-\widetilde{x}_{i}\right]^{2}}$, where $x_{i}(t)$ and $\widetilde{x}_{i}$ are the coordinates of $C_{\alpha}$ atom of residue $i$ at time-step $t$, and their average over $T$ time-steps, respectively.

Supplementary Materials: The following are available online at www.mdpi.com/2072-6651/9/3/109/s1, Table S1: Complementary primers used in mutagenesis, Figure S1: Hydropathy profile of the hydrophobic (HP) region from CyaA-Hly (blue line) compared with three highly-active RTX cytolysins i.e., HlyA, ApxIA and LtxA (green lines), Figure S2: SDS-PAGE (12\% gel) of the soluble lysates extracted from E. coli harboring different plasmids; $p E T-17 b$ vector, $\mathrm{pCyaAC}-\mathrm{PF} / \mathrm{H}_{6}(\mathrm{Wt})$ and $\mathrm{pCyaAC}-\mathrm{PF} / \mathrm{H}_{6}$ with mutations (Q574K, Q574R, Q574E, E581K, E581R and E581Q).

Acknowledgments: We are very grateful to Prof. Michael Mayer (University of Michigan, USA) for providing facilities in planar lipid bilayer studies. A Royal Golden Jubilee Ph.D. Scholarship (to Chattip Kurehong) is gratefully acknowledged. This work was supported in part by grants from the Thailand Research Fund (BRG-58-8-0002 and MRG5680023) and Mahidol University (MU 49/2559) and Higher Educational Research Promotion, Office of the Higher Education Commission (SURDI-CHE 57/01/011 to B.P.).

Author Contributions: Chattip Kurehong, Chalermpol Kanchanawarin, and Chanan Angsuthanasombat conceived and designed the experiments. Chattip Kurehong performed the experiments and wrote the manuscript. Chattip Kurehong, Chalermpol Kanchanawarin, and Chanan Angsuthanasombat analyzed the data. Chattip Kurehong, Chalermpol Kanchanawarin, Busaba Powthongchin, Panchika Prangkio, Gerd Katzenmeier, and Chanan Angsuthanasombat reviewed and edited the manuscript.

Conflicts of Interest: The authors declare no conflict of interest.

\section{Abbreviations}

The following abbreviations are used in this manuscript:

CyaA adenylate cyclase-hemolysin toxin

DiPhyPC 1,2-diphytanoyl-sn-glycero-3-phosphocholine

DMPC 1,2 dimyristoyl-sn-glycero-3-phosphocholine

MD molecular dynamics

$\mathrm{Ni}^{2+}$-NTA nickel-nitrilotriacetic acid

PLBs planar lipid bilayers

RTX Repeat-in-Toxin

$\mathrm{VH} / \mathrm{V}_{\mathrm{H}} \mathrm{H} \quad$ variable heavy chain domain

\section{References}

1. Bart, M.J.; Harris, S.R.; Advani, A.; Arakawa, Y.; Bottero, D.; Bouchez, V.; Cassiday, P.K.; Chiang, C.-S.; Dalby, T.; Fry, N.K.; et al. Global population structure and evolution of Bordetella pertussis and their relationship with vaccination. mBio 2014. [CrossRef] [PubMed]

2. Warfel, J.M.; Zimmerman, L.I.; Merkel, T.J. Acellular pertussis vaccines protect against disease but fail to prevent infection and transmission in a nonhuman primate model. Proc. Natl. Acad. Sci. USA 2014, 111, 787-792. [CrossRef] [PubMed]

3. Lim, A.; Ng, J.K.; Locht, C.; Alonso, S. Protective role of adenylate cyclase in the context of a live pertussis vaccine candidate. Microbes Infect. 2014, 16, 51-60. [CrossRef] [PubMed]

4. Rumbo, M.; Hozbor, D. Development of improved pertussis vaccine. Hum. Vac. Immunother. 2014, 10, 2450-2453. [CrossRef] [PubMed]

5. Cheung, G.Y.; Xing, D.; Prior, S.; Corbel, M.J.; Parton, R.; Coote, J.G. Effect of different forms of adenylate cyclase toxin of Bordetella pertussis on protection afforded by an acullular pertussis vaccine in a murine model. Infect. Immun. 2006, 74, 6797-6805. [CrossRef] [PubMed]

6. Vojtova, J.; Kamanova, J.; Sebo, P. Bordetella adenylate cyclase toxin: A swift saboteur of host defense. Curr. Opin. Microbiol. 2006, 9, 69-75. [CrossRef] [PubMed]

7. Malik, A.A.; Imtong, C.; Sookrung, N.; Katzenmeier, G.; Chaicumpa, W.; Angsuthanasombat, C. Structural characterization of humanized nanobodies with neutralizing activity against the Bordetella pertussis CyaA-hemolysin: Implications for a potential epitope of toxin-protective antigen. Toxins 2016, 8, 99-111. [CrossRef] [PubMed] 
8. Linhartova, I.; Bumba, L.; Masin, J.; Basler, M.; Osicka, R.; Kamanova, J.; Prochazkova, K.; Adkins, I.; Hejnova-Holubova, J.; Sadilkova, L.; et al. RTX proteins: A highly diverse family secreted by a common mechanism. FEMS Microbiol. Rev. 2010, 34, 1076-1112. [CrossRef] [PubMed]

9. Osicka, R.; Osickova, A.; Basar, T.; Guermonprez, P.; Rojas, M.; Leclerc, C.; Sebo, P. Delivery of CD8 ${ }^{+}$T-cell epitopes into major histocompatibility complex class I antigen presentation pathway by Bordetella pertussis adenylate cyclase: Delineation of cell invasive structures and permissive insertion sites. Infect. Immun. 2000, 68, 247-256. [PubMed]

10. Bauche, C.; Chenal, A.; Knapp, O.; Bodenreider, C.; Benz, R.; Chaffotte, A.; Ladant, D. Structural and functional characterization of an essential RTX subdomain of Bordetella pertussis adenylate cyclase toxin. J. Biol. Chem. 2006, 281, 16914-16926. [CrossRef] [PubMed]

11. Pojanapotha, P.; Thamwiriyasati, N.; Powthongchin, B.; Katzenmeier, G.; Angsuthanasombat, C. Bordetella pertussis CyaA-RTX subdomain requires calcium ions for structural stability against proteolytic degradation. Protein Expr. Purif. 2011, 75, 127-132. [CrossRef] [PubMed]

12. Bumba, L.; Masin, J.; Mecek, P.; Wald, T.; Motlova, L.; Bibova, I.; Klimova, N.; Bednarovo, L.; Veverka, V.; Svergun, D.I.; et al. Calcium-driven folding of RTX domain $\beta$-rolls ratchets translocation of RTX proteins through type I secretion ducts. Mol. Cell 2016, 62, 47-62. [CrossRef] [PubMed]

13. Hackett, M.; Guo, L.; Shabanowitz, J.; Hunt, D.F.; Hewlett, E.L. Internal lysine palmitoylation in adenylate cyclase toxin from Bordetella pertussis. Science 1994, 266, 433-435. [CrossRef] [PubMed]

14. Thamwiriyasati, N.; Powthongchin, B.; Kittiworakarn, J.; Katzenmeier, G.; Angsuthanasombat, C. Esterase activity of Bordetella pertussis CyaC-acyltransferase against synthetic substrates: Implications for catalytic mechanism in vivo. FEMS Microbiol. Lett. 2010, 340, 183-190. [CrossRef] [PubMed]

15. Guermonprez, P.; Khelef, N.; Blouin, E.; Rieu, P.; Ricciardi-Castagnoli, P.; Guiso, N.; Ladant, D.; Leclerc, C. The adenylate cyclase toxin of Bordetella pertussis binds to target cells via the $\alpha_{M} \beta_{2}$ integrin (CD11b/CD18). J. Exp. Med. 2001, 193, 1035-1044. [CrossRef] [PubMed]

16. Khelef, N.; Guiso, N. Induction of macrophage apoptosis by Bordetella pertussis adenylate cyclase-hemolysin. FEMS Microbiol. Lett. 1995, 134, 27-32. [CrossRef] [PubMed]

17. Masin, J.; Basler, M.; Knapp, O.; El-Azami-El-Idrissi, M.; Maier, E.; Konopasek, I.; Benz, R.; Leclerc, C.; Sebo, P. Acylation of lysine 860 allows tight binding and cytotoxicity of Bordetella adenylate cyclase on CD11b-expressing cells. Biochemistry 2005, 44, 12759-12766. [CrossRef] [PubMed]

18. Meetum, K.; Imtong, C.; Katzenmeier, G.; Angsuthanasombat, C. Acylation of the Bordetella pertussis CyaA-hemolysin: Functional implications for efficient membrane insertion and pore formation. Biochim. Biophys. Acta-Biomembr. 2017, 1859, 312-318. [CrossRef] [PubMed]

19. Sakamoto, H.; Bellalou, J.; Sebo, P.; Ladant, D. Bordetella pertussis adenylate cyclase toxin. Structural and functional independence of the catalytic and hemolytic activities. J. Biol. Chem. 1992, 267, 13598-13602. [PubMed]

20. Vojtova, J.; Kofronova, O.; Sebo, P.; Benada, O. Bordetella adenylate cyclase toxin induces a cascade of morphological changes of sheep erythrocytes and localizes into clusters in erythrocyte membranes. Microsc. Res. Tech. 2006, 69, 119-129. [CrossRef] [PubMed]

21. Powthongchin, B.; Angsuthanasombat, C. High level of soluble expression in Escherichia coli and characterisation of the CyaA pore-forming fragment from a Bordetella pertussis Thai clinical isolate. Arch. Microbiol. 2008, 189, 169-174. [CrossRef] [PubMed]

22. Pandit, R.A.; Meetum, K.; Suvarnapunya, K.; Katzenmeier, G.; Chaicumpa, W.; Angsuthanasombat, C. Isolated CyaA-RTX subdomain from Bordetella pertussis: Structural and functional implications for its interaction with target erythrocyte membranes. Biochem. Biophys. Res. Commun. 2015, 466, 76-81. [CrossRef] [PubMed]

23. Powthongchin, B.; Angsuthanasombat, C. Effects on haemolytic activity of single proline substitutions in the Bordetella pertussis CyaA pore-forming fragment. Arch. Microbiol. 2009, 191, 1-9. [CrossRef] [PubMed]

24. Juntapremjit, S.; Thamwiriyasati, N.; Kurehong, C.; Prangkio, P.; Shank, L.; Powthongchin, B.; Angsuthanasombat, C. Functional importance of the Gly cluster in transmembrane helix 2 of the Bordetella pertussis CyaA-hemolysin: Implications for toxin oligomerization and pore formation. Toxicon 2015, 106, 14-19. [CrossRef] [PubMed] 
25. Kurehong, C.; Powthongchin, B.; Thamwiriyasati, N.; Angsuthanasombat, C. Functional significance of the highly conserved Glu ${ }^{570}$ in the putative pore-forming helix 3 of the Bordetella pertussis haemolysin toxin. Toxicon 2011, 57, 897-903. [CrossRef] [PubMed]

26. Kurehong, C.; Kanchanawarin, C.; Powthongchin, B.; Katzenmeier, G.; Angsuthanasombat, C. Membrane-pore forming characteristics of the Bordetella pertussis CyaA-hemolysin domain. Toxins 2015, 7, 1486-1496. [CrossRef] [PubMed]

27. Masin, J.; Osickova, A.; Sukova, A.; Fiser, R.; Halada, P.; Bumba, L.; Linhartova, I.; Osicka, R.; Sebo, P. Negatively charged residues of the segment linking the enzyme and cytolysin moieties restrict the membrane-permeabilizing capacity of adenylate cyclase toxin. Sci. Rep. 2016. [CrossRef] [PubMed]

28. Kyte, J.; Doolittle, R.F. A simple method for displaying the hydropathic character of a protein. J. Mol. Biol. 1982, 157, 105-132. [CrossRef]

29. Benz, R.; Maier, E.; Ladant, D.; Ullmann, A.; Sebo, P. Adenylate cyclase toxin (CyaA) of Bordetella pertussis: Evidence for the formation of small ion-permeable channels and comparison with HlyA of Escherichia coli. J. Biol. Chem. 1994, 269, 27231-27239. [PubMed]

30. Maier, E.; Reinhard, N.; Benz, R.; Frey, J. Channel-forming activity and channel size of the RTX toxins ApxI, ApxII, and ApxIII of Actinobacillus pleuropneumoniae. Infect. Immun. 1996, 64, 4415-4423. [PubMed]

31. Menestrina, G.; Moser, C.; Pellet, S.; Welch, R. Pore-formation by Escherichia coli hemolysin (HlyA) and other members of the RTX toxins family. Toxicology 1994, 87, 249-267. [CrossRef]

32. Kato, N.; Akai, M.; Zulkifli, L.; Matsuda, N.; Kato, Y.; Goshima, S.; Hazama, A.; Yamagami, M.; Guy, H.R.; Uozumi, N. Role of positively charged amino acids in the M2D transmembrane helix of Ktr/Trk/HKT type cation transporters. Channels 2007, 1, 161-171. [CrossRef] [PubMed]

33. Muller, D.J.; Wu, N.; Palczewski, K. Vertebrate membrane proteins: Structure, function, and insights from biophysical approaches. Pharmacol. Rev. 2008, 60, 43-78. [CrossRef] [PubMed]

34. Phale, P.S.; Philippsen, A.; Widmer, C.; Phale, V.P.; Rosenbusch, J.P.; Schirmer, T. Role of charged residues at the OmpF porin channel constriction probed by mutagenesis and simulation. Biochemistry 2001, 40, 6319-6325. [CrossRef] [PubMed]

35. Ehrmann, I.E.; Gray, M.C.; Gordon, V.M.; Gray, L.S.; Hewlett, E.L. Hemolytic activity of adenylate cyclase toxin from Bordetella pertussis. FEBS Lett. 1991, 278, 79-83. [PubMed]

36. Juntadech, T.; Kanintronkul, Y.; Kanchanawarin, C.; Katzenmeier, G.; Angsuthanasombat, C. Importance of polarity of the $\alpha 4-\alpha 5$ loop residue-Asn ${ }^{166}$ in the pore-forming domain of the Bacillus thuringiensis Cry4Ba toxin: Implications for ion permeation and pore opening. Biochim. Biophys. Acta-Biomembr. 2014, 1838, 319-327. [CrossRef] [PubMed]

37. Sriwimol, W.; Aroonkesorn, A.; Sakdee, S.; Kanchanawarin, C.; Uchihashi, T.; Ando, T.; Angsuthanasombat, C. Potential pre-pore trimer formation by the Bacillus thuringiensis mosquito-specific toxin: Molecular insights into a critical prerequisite of membrane-bound monomers. J. Biol. Chem. 2015, 290, 20793-20803. [CrossRef] [PubMed]

38. Larkin, M.A.; Blackshields, G.; Brown, N.P.; Chenna, R.; McGettigan, P.A.; McWilliam, H.; Valentin, F.; Wallace, I.M.; Wilm, A.; Lopez, R.; et al. Clustal W and Clustal X version 2.0. Bioinformatics 2007, 23, 2947-2948. [CrossRef] [PubMed]

39. Schwede, T.; Kopp, J.; Guex, N.; Peitsch, M.C. SWISS-MODEL: An automated protein homology-modeling server. Nucl. Acids Res. 2003, 31, 3381-3385. [CrossRef] [PubMed]

40. Lindahl, E.; Azuara, C.; Koehl, P.; Delarue, M. NOMAD-Ref: Visualization, deformation and refinement of macromolecular structures based on all-atom normal mode analysis. Nucl. Acids Res. 2006, 34, W52-W56. [CrossRef] [PubMed]

41. Pierce, B.G.; Wiehe, K.; Hwang, H.; Kim, B.H.; Vreven, T.; Weng, Z. ZDOCK server: interactive docking prediction of protein-protein complexes and symmetric multimers. Bioinformatics 2014, 30, 1771-1773. [CrossRef] [PubMed]

42. Wu, E.L.; Cheng, X.; Jo, S.; Rui, H.; Song, K.C.; Davila-Contreras, E.M.; Qi, Y.; Lee, J.; Monje-Galvan, V.; Venable, R.M.; et al. CHARMM-GUI Membrane Builder toward realistic biological membrane simulations. J. Comput. Chem. 2014, 35, 1997-2004. [CrossRef] [PubMed]

43. Humphrey, W.; Dalke, A.; Schulten, K. VMD: Visual molecular dynamics. J. Mol. Graph. 1996, 14, 33-38. [CrossRef] 
44. Phillips, J.C.; Braun, R.; Wang, W.; Gumbart, J.; Tajkhorshid, E.; Villa, E.; Chipot, C.; Skeel, R.D.; Kale, L.; Schulten, K. Scalable molecular dynamics with NAMD. J. Comput. Chem. 2005, 26, 1781-1802. [CrossRef] [PubMed]

45. MacKerell, A.D., Jr.; Bashford, D.; Bellott, M.; Dunbrack, R.L., Jr.; Evanseck, J.D.; Field, M.J.; Fischer, S.; Gao, J.; Guo, H.; Ha, S.; et al. All-atom empirical potential for molecular modeling and dynamics studies of proteins. J. Phys. Chem. 1998, 102, 3586-3616. [CrossRef] [PubMed]

46. Taveecharoenkool, T.; Angsuthanasombat, C.; Kanchanawarin, C. Combined molecular dynamics and continuum solvent studies of the pre-pore Cry4Aa trimer suggest its stability in solution and how it may form pore. PMC Biophys. 2010. [CrossRef] [PubMed]

(C) 2017 by the authors. Licensee MDPI, Basel, Switzerland. This article is an open access article distributed under the terms and conditions of the Creative Commons Attribution (CC BY) license (http:/ / creativecommons.org/licenses/by/4.0/). 